\title{
Synaptonemal complex formation in two allohexaploid Festuca species and a pentaploid hybrid
}

\author{
HUW M. THOMAS* \& B. J. THOMAS \\ AFRC Institute of Grassland and Environmental Research, Plas Gogerddan, Aberystwyth, Wales, SY23 3EB, U.K.
}

\begin{abstract}
Festuca arundinacea and Festuca gigantea are allohexaploids $(2 n=6 x=42)$, that have bivalent pairing at metaphase I of meiosis. Analysis of the synaptonemal complexes of these species and of a pentaploid hybrid between $F$. gigantea and tetraploid Lolium perenne showed that initial synapsis in the allohexaploids is mostly between homologous chromosomes though some multivalents are formed, but in the hybrid most of the chromosomes associate as multivalents. It is concluded that the mechanism controlling bivalent formation in these species acts mainly at zygotene by restricting pairing to homologous chromosomes, but also at pachytene preventing cross-overs in the small number of homoeologous associations that have occurred. In the hybrid, where pairing control is ineffective, the mechanism fails at both stages.
\end{abstract}

Keywords: Festuca, hexaploid, pairing control, pentaploid, synaptonemal complex.

\section{Introduction}

The grass genus Festuca comprises a large number of diverse species forming a polyploid series from diploid to decaploid (Borrill et al., 1977 and references therein), and all the species studied have bivalent pairing at metaphase I of meiosis (Thomas et al., 1982 and references therein). $F$. arundinacea is an allohexaploid species $(2 n=6 x=42)$; its inheritance is disomic (Lewis et al., 1980) and bivalent pairing has been shown to be under genetic control by a gene(s) on one pair of chromosomes (Jauhar, 1975). The gene is hemizygous ineffective: therefore, in hybrids where the three $F$. arundinacea genomes are in the haploid state, the homoeologous chromosomes can freely pair to form bivalents and multivalents (e.g. Evans \& Aung, 1986). $F$. gigantea is also a bivalent forming allohexaploid and the diploidization mechanism appears similar in the two species, though there may be less affinity between the constituent genomes of $F$. gigantea than between those of $F$. arundinacea (Morgan et al., 1988).

Chromosome pairing at prophase I of meiosis has, to date, been examined by synaptonemal complex analysis in two other allohexaploids, namely Triticum aestivum (Hobolth, 1981; Jenkins, 1983; Holm, 1986; Holm \& Wang, 1988) and Avena sativa (Jones et al., 1989).

*Correspondence.
These studies show that in hexaploid wheat there is some multivalent pairing at zygotene even when the $\mathrm{Ph}$ locus is present but it may be corrected by pachytene. No multivalents were observed in the small number of Avena meiocytes analysed at zygotene. Diploidization in Avena is apparently achieved by limiting initial synapsis to homologous chromosomes only. The present study is part of a programme to understand the mechanism of pairing control in the Lolium-Festuca complex and describes SC formation in prophase nuclei of the two allohexaploid species $F$. arundinacea and $F$. gigantea and a pentaploid hybrid $L$. perenne $(4 x) \times F$. gigantea .

\section{Materials and methods}

Four genotypes of $F$. arundinacea were used from the WPBS accession Bn 496, and three genotypes of $F$. gigantea from accession Bs 4088. Two hybrid genotypes were studied from crosses of synthetic auto-tetraploid L. perenne $\mathrm{Ba} 9954$ and $\mathrm{Ba} 10794$ with $F$. gigantea Bs 3781 .

Anthers were taken from inflorescences partly emerged from the leaf sheath and the stage determined by squashing one anther in 1 per cent aceto-carmine. The methods of spreading and staining the SCs are described by Thomas (1990).

Electron micrographs were taken on a Jeol $100 \mathrm{~S}$ T.E.M. at a magnification of $3 \mathrm{~K}$ for 10 of the $12 F$. 
arundinacea spreads and for 3 of the $18 \mathrm{~F}$. gigantea spreads. The remaining images of the hexaploids and all those of the hybrid nuclei were recorded at $2 \mathrm{~K}$. The photographs were enlarged three-fold when printed giving final magnifications of approximately $9 \mathrm{~K}$ or $6 \mathrm{~K}$, the exact magnification being determined for each nucleus by measurements of accompanying images of a cross grating (2160 lines per $\mathrm{mm}$ ). The lengths of axial elements were measured by a digitizer tablet and microcomputer, while the distances between axial elements were measured with a micrometer eye-piece. The width measurements were only taken from those prints with magnifications of $9 \mathrm{~K}$ as measurements at lower magnifications were not considered sufficiently accurate.

\section{Results}

\section{F. arundinacea}

Table 1 summarizes the analysis of 10 nuclei at zygotene, pachytene and diplotene. The nuclei were classified under these stages by the extent of pairing and on the general morphology of the axial elements and SCs. The two nuclei considered to be at late zygotene have 77 and 79 per cent of the axial element length paired with some bivalents less than 50 per cent paired. The four nuclei judged to be at pachytene are almost fully paired with the limited amount of asynapsis mainly associated with the pairing partner exchanges and at a small number of telomeres, some of which are of unequal length. In those nuclei classified as diplotene one-third of the axial elements are disintegrating or are being repulsed. The percentage of paired axial elements in Table 1 includes these SCs.

Table 1 Summary of the analysis of 10 nuclei of $F$. arundinacea

\begin{tabular}{lcll}
\hline Nucleus & Pairing $(\%)$ & $\begin{array}{l}\text { Element length } \\
(\mu \mathrm{m})\end{array}$ & $\begin{array}{l}\text { Pairing } \\
\text { configurations }\end{array}$ \\
\cline { 2 - 4 }$a \mathrm{EZ7}$ & $<10$ & & \\
$a \mathrm{LZ9}$ & 77 & 1258 & $21 \mathrm{II}$ \\
$a \mathrm{LZ1}$ & 79 & 1585 & $21 \mathrm{II}$ \\
$a \mathrm{P} 15$ & 92 & 1227 & $1 \mathrm{VI}, 1 \mathrm{IV}, 16 \mathrm{II}$ \\
$a \mathrm{P} 10$ & 93 & 1082 & $1 \mathrm{IV}, 19 \mathrm{II}$ \\
$a \mathrm{P} 4$ & 97 & 1091 & $21 \mathrm{II}$ \\
$a \mathrm{P} 5$ & 98 & 1128 & $21 \mathrm{II}$ \\
$a \mathrm{D} 11$ & 98 & 1142 & $2 \mathrm{IV}, 17 \mathrm{II}$ \\
$a \mathrm{D} 2$ & 99 & 1301 & $21 \mathrm{II}$ \\
$a \mathrm{D} 12$ & 100 & 1062 & $2 \mathrm{IV}, 17 \mathrm{II}$ \\
\hline
\end{tabular}

$\mathrm{EZ}=$ early zygotene, $\mathrm{LZ}=$ late zygotene, $\mathrm{P}=$ pachytene, $\mathrm{D}=$ diplotene.
The earliest zygotene nucleus was estimated to have less than 10 per cent of the axial element length paired, though total length was not measured. Pairing at pachytene and diplotene is almost complete. The total axial element length of the late zygotene nucleus $a \mathrm{LZ} 1$ is $1585 \mu \mathrm{m}$ but by pachytene the total length is reduced to $1062 \mu \mathrm{m}$. Axial element length increases again in some diplotene nuclei though this may be a distortion caused by the fragmenting of the SCs. The width of the SCs varies somewhat, both within and between nuclei (102-132 $\mathrm{nm}$ at zygotene, $124-145 \mathrm{~nm}$ at pachytene, $152 \mathrm{~nm}$ at diplotene) but there is even greater variation in the distances between axial elements when aligned prior to synapsis (224-643 nm) and at diplotene when the SCs begin to break down, axial elements may be fused or be $400 \mathrm{~nm}$ apart. Pairing configurations are predominantly bivalents though multivalents are found.

At early zygotene the axial elements are somewhat granular in nature and difficult to trace; in consequence only one nucleus was analysed and this not completely so. The 14 SCs recorded have a total length of $103 \mu \mathrm{m}$. Additionally, $206 \mu \mathrm{m}$ of axial element is aligned.

At late zygotene, axial elements are continuous and easier to trace. The two nuclei analysed consist entirely of SCs and aligned axial elements with up to 5 SC regions per bivalent. While some breaks in elements in $a$ LZ1 mean that we could not exclude the possibility of one quadrivalent, in aLZ9 there were clearly 21 bivalents. Three bivalents are fully paired and three others more than 90 per cent paired with one SC extending from one telomere with the other telomere unpaired. The remaining bivalents are between 42 and 85 per cent paired with 3 to 5 individual SC regions each.

The four nuclei judged to be at pachytene are 92-98 per cent paired. Two nuclei contain multivalents. In aP15 some of the axial elements associated with the pairing exchanges are broken but as there are 16 bivalents and clearly two multivalents the interpretation of one quadrivalent and one hexavalent is the only one possible. In $a \mathrm{P} 10$ there is one quadrivalent and 19 bivalents. The quadrivalent is abnormal with one unsynapsed arm and one heterologous, reverse paired SC (Fig. 1). The two homologous pairs can be identified by their lengths. Axes $\mathrm{AC}$ and $\mathrm{AB}$ are $30 \mu \mathrm{m}$ and $34 \mu \mathrm{m}$ long while $\mathrm{CD}$ and $\mathrm{BE}$ are $21 \mu \mathrm{m}$ and $23 \mu \mathrm{m}$, respectively.

The SCs in the three early diplotene nuclei seem to be disintegrating. The axial elements have not only moved apart but elsewhere the SC has collapsed inwards (Fig. 2a). The most striking feature of $a \mathrm{D} 2$ is the many breaks in the SCs (Fig. 2b) which is perhaps a contributing factor to the increase in total axial element 

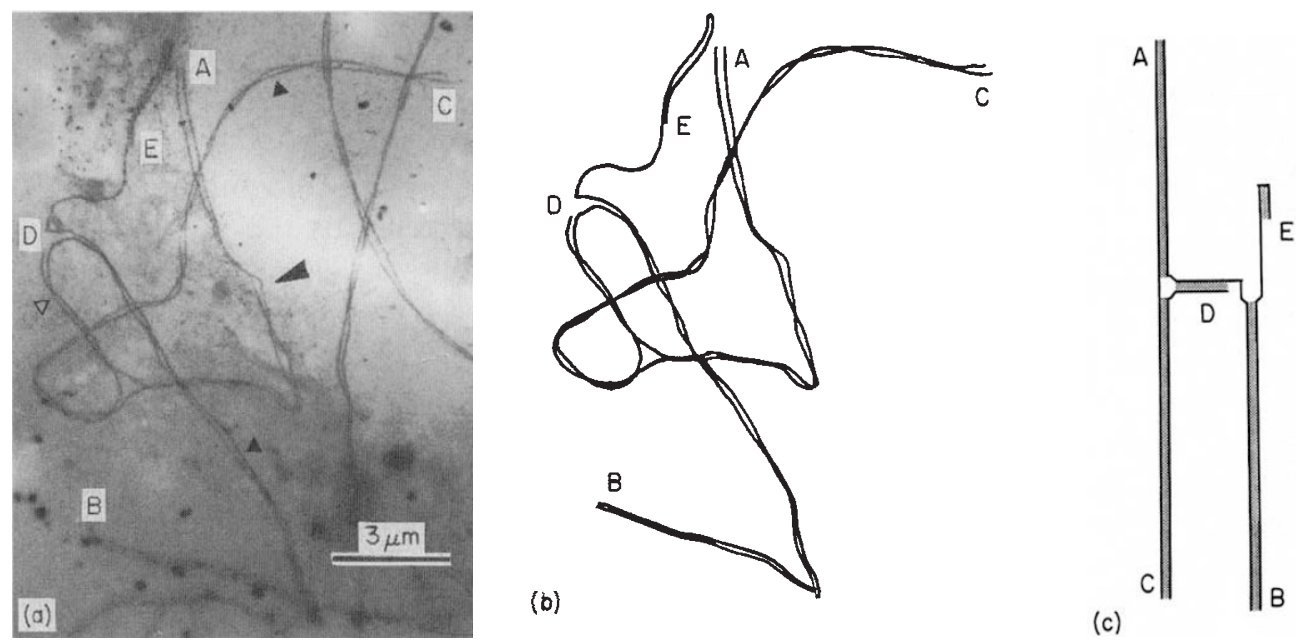

Fig. 1 (a) Part of a pachytene nucleus of $F$. arundinacea with a quadrivalent involving two homologous pairs $\mathrm{AB} / \mathrm{AC}$ and $\mathrm{CD} /$ BE. (b) Tracing of the quadrivalent in (a). (c) Diagram of the quadrivalent. Large arrowhead indicates a SC between homologous axial elements, solid triangles indicate homoeologous or non-homologous pairing, the open triangle indicates a SC with reverse polarity. Bar represents $3 \mu \mathrm{m}$.

Fig. 2 Details from two diplotene nuclei of $F$. arundinacea. (a) SCs have collapsed inwards (arrows). (b) axial elements have moved apart and are discontinuous. Bar represents $2 \mu \mathrm{m}$.
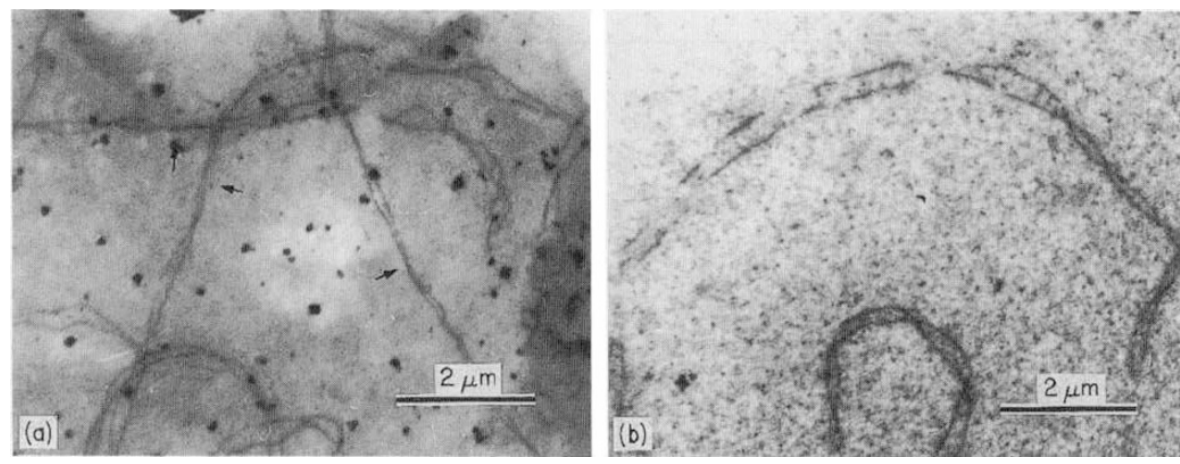

length. There are two quadrivalents in each of $a \mathrm{D} 11$ and $a \mathrm{D} 12$.

As Table 1 shows, there are multivalents in four of the 10 nuclei analysed in $F$. arundinacea comprising four quadrivalents and one hexavalent.

\section{F. gigantea}

The analysis of 18 nuclei is summarized in Table 2 . Seventeen nuclei are at pachytene and one at diplotene. All nuclei are at least 98 per cent paired, though there is a more than two-fold difference in total axial element length between nuclei, from $1002 \mu \mathrm{m}$ to $2169 \mu \mathrm{m}$. In 15 pachytene nuclei there are 21 bivalents. There is one quadrivalent in each of the other two nuclei; in nucleus $g \mathrm{P} 5$ there is asynapsis at the pairing partner exchange amounting to 18 per cent of the axial element length but the quadrivalent in nucleus $g \mathrm{P} 1$ is fully paired, though this includes a foldback SC (Fig. 3). The two homologous pairs in this quadrivalent cannot be identified by the individual lengths of the axial elements as there is only a $3 \mu \mathrm{m}$ difference between the longest and shortest axis.

Although there are breaks in the SCs in the diplotene nucleus $g \mathrm{D} 26$ it could with confidence be analysed as 21 bivalents. In common with the diplotene nuclei of $F$. arundinacea, the SCs show signs of disintegration and increase in the total length.

\section{The pentaploid hybrid, L. perenne $(4 x) \times \mathrm{F}$. gigantea}

The eight nuclei analysed cover stages from early zygotene to diplotene. Total axial element length varies from $2168 \mu \mathrm{m}$ at early zygotene to $988 \mu \mathrm{m}$ at diplotene, with pairing varying from 27 per cent to 85 per cent. However, in a pentaploid hybrid the equivalent of one chromosome in each homoeologous group will be without a partner; therefore, assuming that the five chromosomes within each homoeologous group are similar in length, the maximum homologous and 
Table 2 Summary of the analysis of 18 nuclei of F. gigantea

\begin{tabular}{|c|c|c|c|}
\hline Nucleus & Pairing (\%) & $\begin{array}{l}\text { Element length } \\
(\mu \mathrm{m})\end{array}$ & $\begin{array}{l}\text { Pairing } \\
\text { configurations }\end{array}$ \\
\hline$g \mathrm{P} 5$ & 98 & 1203 & $1 \mathrm{IV}, 19 \mathrm{II}$ \\
\hline$g \mathrm{P} 2$ & 99 & 1152 & $21 \mathrm{II}$ \\
\hline$g \mathrm{P} 10$ & 99 & 1140 & $21 \mathrm{II}$ \\
\hline gP1 & 100 & 2169 & $1 \mathrm{IV}, 19 \Pi$ \\
\hline$g \mathrm{P} 11$ & 100 & 1741 & $21 \mathrm{II}$ \\
\hline$g \mathrm{P} 24$ & 100 & 1447 & $21 \mathrm{II}$ \\
\hline$g \mathrm{P} 8$ & 100 & 1422 & $21 \mathrm{II}$ \\
\hline gP14 & 100 & 1349 & $21 \mathrm{II}$ \\
\hline gP15 & 100 & 1297 & $21 \mathrm{II}$ \\
\hline$g \mathrm{P} 23$ & 100 & 1243 & $21 \mathrm{II}$ \\
\hline gP4 & 100 & 1235 & $21 \mathrm{II}$ \\
\hline gP6 & 100 & 1228 & $21 \mathrm{II}$ \\
\hline$g \mathrm{P} 13$ & 100 & 1138 & $21 \mathrm{II}$ \\
\hline gP7 & 100 & 1120 & $21 \mathrm{II}$ \\
\hline $\mathrm{gP} 12$ & 100 & 1081 & $21 \mathrm{II}$ \\
\hline gP9 & 100 & 1017 & $21 \mathrm{II}$ \\
\hline gP3 & 100 & 1002 & $21 \mathrm{II}$ \\
\hline$g \mathrm{D} 26$ & 100 & 1389 & $21 \mathrm{II}$ \\
\hline
\end{tabular}

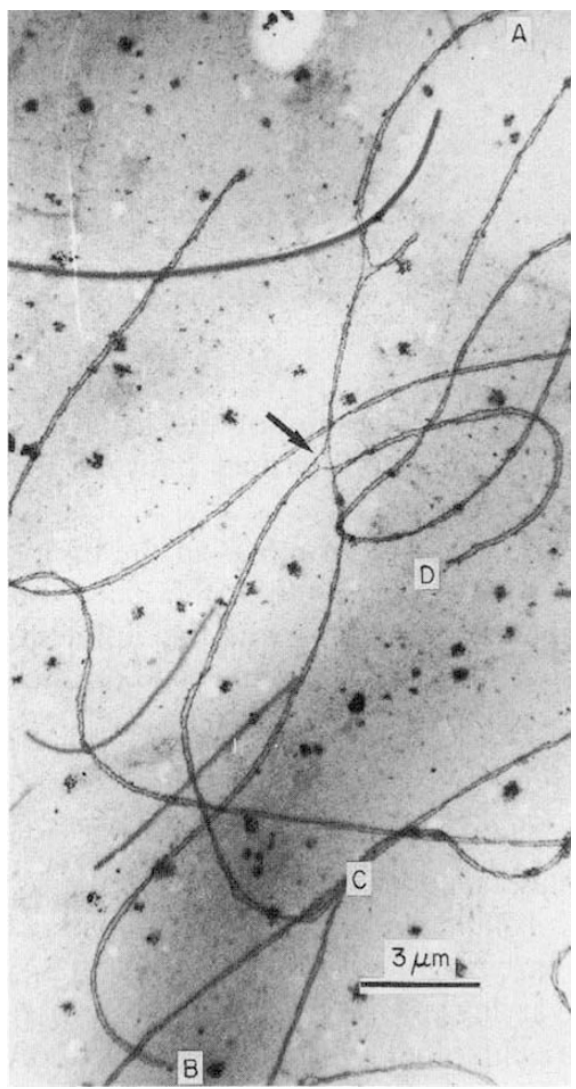

Fig. 3 Part of a pachytene nucleus of $F$. gigantea showing a quadrivalent. Arrow indicates pairing partner exchange. Bar represents $3 \mu \mathrm{m}$. homoeologous pairing will be about 80 per cent of the total axial element length. On this basis the pachytene nuclei are fully paired (Table 3). Pairing has in fact proceeded beyond that necessary for synapsis of homologous and homoeologous regions; heterologous pairing is detected by the presence of associations of more than five axial elements.

In the earliest zygotene nucleus ( $h \mathrm{Z} 3)$ one third of homologous plus homoeologous length is paired and the SCs are interspersed with lengths of closely aligned axial elements. One association of four axial elements and three associations of three axial elements can be seen but not all axial elements can be traced through their entire lengths. Therefore there may be some pairing partner exchanges undetected. In nucleus $h \mathrm{Z} 2$ there are at least three fully paired bivalents but also extensive multivalent formation though this cannot be fully analysed. In $h \mathrm{Z} 4$ there are at least four fully paired bivalents, with one pentavalent and three quadrivalents which could be parts of a larger multivalent. In $h \mathrm{Z} 1$ the multivalent formation is complex with several cases of interlocking, and axial elements are generally tangled.

In pachytene nucleus $h \mathrm{P} 13$ there are at least seven fully paired bivalents, while the remaining axial elements are involved in multivalent formation. There is one association of at least eight axial elements and one of six, and these two groups are almost certainly associated with each other. An inversion type loop is present in a quadrivalent (Fig. 4). There are mismatched telomeres and also telomeric associations between SCs and unpaired axial elements. In three instances unpaired axial elements appear split and double in nature. Nucleus $h \mathrm{P} 14$ has four bivalents while the remaining axial elements appear to be associated together in one complex multivalent. There are nine bivalents in $h \mathrm{P} 12$, the remaining axial elements forming one large multivalent (Fig. 5).

Table 3 Summary of the analysis of 8 nuclei of the pentaploid hybrid $L$. perenne $(4 x) \times F$. gigantea

\begin{tabular}{lll}
\hline Nucleus & $\begin{array}{l}\text { Pairing } \\
\text { (\% of total length) }\end{array}$ & $\begin{array}{l}\text { Element length } \\
(\mu \mathrm{m})\end{array}$ \\
\hline$h \mathrm{Z3}$ & 27 & 2168 \\
$h \mathrm{Z} 2$ & 48 & 1392 \\
$h \mathrm{Z} 4$ & 58 & 1500 \\
$h \mathrm{Z} 1$ & 59 & 1278 \\
$h \mathrm{P} 14$ & 76 & 1074 \\
$h \mathrm{P} 12$ & 83 & 1036 \\
$h \mathrm{P} 13$ & 85 & 1208 \\
$h \mathrm{D} 11$ & 77 & 988 \\
\hline
\end{tabular}



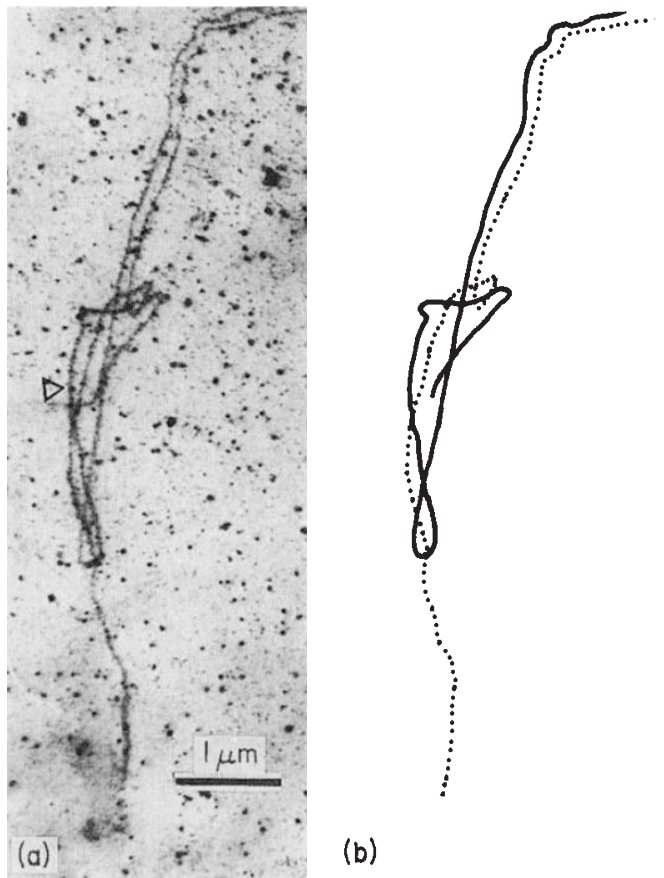

Fig. 4 (a) Part of a pachytene nucleus of the pentaploid hybrid showing an inversion-type loop. The open triangle indicates the SC with reverse polarity. (b) Interpretive drawing of (a). Bar represents $1 \mu \mathrm{m}$.
The diplotene nucleus $h \mathrm{D} 11$ is characterised by extensive breakages in both SCs and unpaired axial elements; many broken ends of SCs appear forked and are probably points of pairing partner exchange. The configurations analysed consist of one septavalent, hexavalent and quadrivalent, three trivalents and two bivalents, and some of the multivalents together with several fragments probably form a large complex multivalent. The SCs are not disintegrating in the way seen in the diplotene nuclei of the hexaploids.

\section{Discussion}

Bivalent pairing is achieved in both T. aestivum and $A$. sativa by a mechanism that is under genetic control. When the genes responsible for the mechanism are absent or suppressed, pairing occurs between homoeologous chromosomes. Homoeologous chromosomes are therefore capable of pairing with each other; any distinction between them, whether it be structural or positional is not in itself sufficient to prevent them from pairing (Riley, 1960; Sears, 1976; Rajhathy \& Thomas, 1972; Gauthier \& McGinnis, 1968; Leggett, 1977).

The homoeologous chromosomes in the Festuca hexaploids are also capable of pairing with each other when the pairing control mechanism is ineffective. In $F$. arundinacea the gene(s) responsible is on one pair of chromosomes and when only one of the pair is missing,
Fig. 5 A pachytene nucleus of the pentaploid hybrid with extensive multivalent formation. Bar represents $5 \mu \mathrm{m}$.

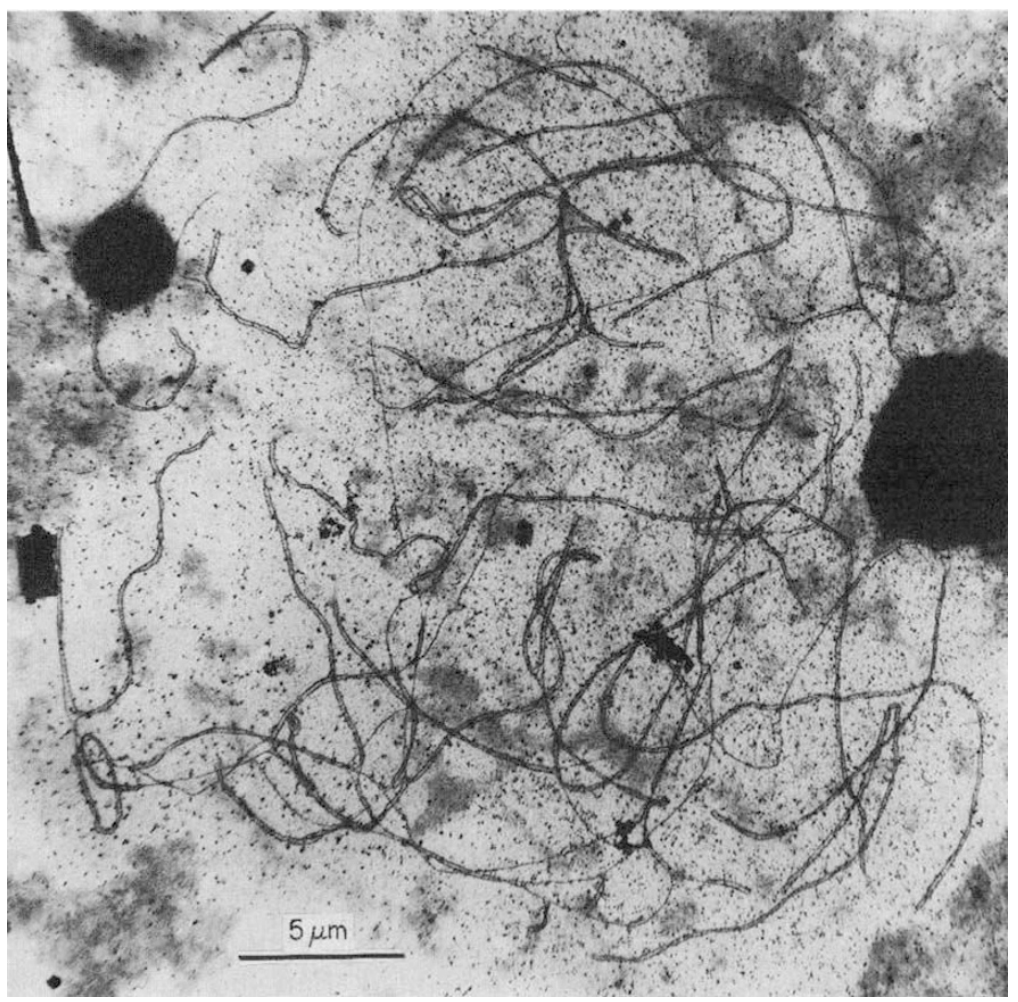


multivalents are formed (Jauhar, 1975). The mechanism is hemizygous ineffective. This condition prevails in hybrids, where the $F$. arundinacea genomes are in the haploid state. In hybrids with $L$. multiflorum and $L$. perenne, pairing occurs between the homoeologous Festuca chromosomes and with the Lolium chromosomes (Kleijer, 1984; Evans \& Aung, 1986). Although the monosomic has not been found in $F$. gigantea, homoeologous pairing in hybrids again demonstrates that the control mechanism is hemizygous ineffective (Morgan et al., 1988).

SC analysis of a single zygotene nucleus of $A$. sativa showed there were no multivalents. The same was true of two zygotene nuclei in the tetraploid, A. maroccana. Also, no multivalents were found in the pachytene nuclei of either species (Jones et al., 1989). The authors concluded that the pairing control mechanism works in Avena by limiting the initial synapsis to homologous chromosomes. At zygotene in wheat, Holm (1986) found some multivalents, though pairing was mainly as bivalents. The number of multivalents decreased by pachytene but some persisted beyond the stage at which cross-overs are presumed occur. Holm concluded that the control mechanism in wheat operates in three ways: (i) initial synapsis is restricted mainly to homologous partners; (ii) a correction mechanism eliminates some multivalents before cross-over takes place; (iii) cross-overs are prevented in homoeologous associations of surviving multivalents. In wheat plants monosomic for chromosome $5 \mathrm{~B}$ more multivalents were formed than in euploids at zygotene and survived through pachytene, but still cross-overs in homoeologous associations were prevented (Holm, 1988a). However in the nullisomics even more multivalents were formed at zygotene and survived through pachytene, and here cross-overs occurred in homoeologous associations (Holm, 1988b).

The data presented here show that in euploid $F$. arundinace $a$ and $F$. gigantea, pairing during prophase is mainly as bivalents though some multivalents are formed. The number of diplotene nuclei analysed is small, nevertheless in $F$. arundinacea there are four quadrivalents in three nuclei. Therefore, on this limited evidence there does not seem to be a correction of multivalents, but in common with wheat there is a suppression of cross-overs in homoeologous associations as no multivalents are found at metaphase $I$. There is a higher frequency of multivalents during prophase stages in $F$. arundinacea than $F$. gigantea. In nine nuclei of $F$. arundinacea a mean of 2.22 axial elements are involved in multivalents as opposed to 0.44 axial elements in $F$. gigantea. This appears to confirm an earlier interpretation that the constituent genomes of $F$. gigantea are more distantly related than those of F. arundinacea (Morgan et al., 1988).
There is some evidence of structural differentiation between homoeologous chromosomes. In one of the quadrivalents found in $F$. arundinacea (Fig. 1) one homologous pair is 45 per cent longer than the other. The truncated arms of the shorter pair have failed to synapse with each other, perhaps through some constraint caused by the asymmetric nature of the configuration. The SC with reverse polarity suggests that the structural change involved a deletion and an inversion. In the hybrid an inversion-type loop (Fig. 4) may be indicative of an inversion difference between homoeologous chromosomes but both may be examples of heterologous pairing.

As the pairing control mechanism is hemizygous ineffective, analysis of the hybrid between $F$. gigantea and $L$. perenne reveals what happens when chromosomes are released from the restrictions imposed on them in the euploid. In a similar pentaploid hybrid between $F$. gigantea and tetraploid L. multiflorum, Morgan et al. (1988) found mean chromosome associations of 4.26 univalents, 9.64 bivalents, 2.11 trivalents, 0.92 quadrivalents and 0.29 pentavalents per cell at metaphase I. The results of the present SC analysis shows some bivalents together with extensive multivalent formation. At pachytene and diplotene, the presence of associations of more than five axial elements which do not persist to metaphase I demonstrates that in addition to homologous and homoeologous pairing, heterologous pairing also occurs. There seems to be no suppression of homoeologous synapsis when the pairing control gene(s) is in the hemizygous state nor is there any means of preventing crossing-over between homoeologously synapsed chromosome arms. Though there are instances of chiasma formation in heterologously synapsed chromosomes in the LoliumFestuca complex (Thomas et al., 1990), Morgan et al. (1988) did not find associations of more than five chromosomes at metaphase $I$ in the $F$. gigantea $\times L$. multiforum pentaploid hybrid. It may be that the heterologous associations found in the present study did not form until after the initiation of cross-overs, or else the heterologous SCs did not support cross-overs.

The first stage of control of chromosome pairing in Festuca, as in wheat and oats acts at initial synapsis ensuring that most chromosomes are associated as bivalents. This stage of control is however less effective in $F$. arundinacea than in $F$. gigantea or wheat and seems less effective in all three species than in oats. Though there is no evidence of a correction of multivalents in the Festuca species during prophase I, a second stage of control acts to prevent cross-overs between homoeologous chromosomes. Both stages fail when the pairing control gene is in the hemizygous state. 


\section{Acknowledgements}

Thanks to our colleagues W. G. Morgan for kindly providing the plant material, Peter Roberts for processing the electron micrographs and Sanjay Mistry for technical assistance for part of the study.

\section{References}

BORRILL, M., KIRBY, M. AND MORGAN, w. G. 1977. Studies in Festuca. 11. Interrelationships of some putative diploid ancestors of the polyploid broad-leaved fescues. New Phytologist, 78, 661-674.

EVANS, G. M. AND AUNG, T. 1986. The influence of the genotype of Lolium perenne on homoeologous chromosome association in hexaploid Festuca arundinacea. Heredity, 56, 97-103.

GAUTHIER, F. M. AND McGinNIS, R. C. 1968. The meiotic behaviour of a nulli-haploid plant in Avena sativa L. Can. J. Gen. Cytol., 10, 186-189.

новоцтн, Р. 1981. Chromosome pairing in allohexaploid wheat var. Chinese Spring. Transformation of multivalents into bivalents, a mechanism for exclusive bivalent formation. Carlsberg Res. Commun., 46, 129-173.

ноLм, Р. в. 1986. Chromosome pairing and chiasma formation in allohexaploid wheat, Triticum aestivum, analysed by spreading of meiotic nuclei. Carlsberg Res. Commun., 51, 239-294.

ноLм, Р. в. 1988a. Chromosome pairing and synaptonemal complex formation in hexaploid wheat, monosomic for chromosome 5B. Carlsberg Res. Commun., 53, 57-89.

ноLм, Р. в. 1988 b. Chromosome pairing and synaptonemal complex formation in hexaploid wheat, nullisomic for chromosome 5B. Carlsberg Res. Commun., 53, 91-110.

HOLM, P. B. AND WANG. x. 1988. The effect of chromosome 5B on synapsis and chiasma formation in wheat, Triticum aestivum, cv. Chinese Spring. Carlsberg Res. Commun., 53, 191-208.
JENKINS, G. 1983. Chromosome pairing in Triticum aestivum cv. Chinese Spring. Carlsberg Res. Commun., 48, 255-283.

JONES, M., REES, H. AND JENKINS, G. 1989. Synaptonemal complex formation in Avena polyploids. Heredity, 63, 209-219.

JAUHAR, P. P. 1975. Genetic control of diploid-like meiosis in hexaploid tall fescue. Nature, 254, 595-597.

KLEIJER, G. 1984. Cytogenetic studies of crosses between Lolium multiflonim Lam. and Festuca arundinacea Shreb. I. The parents and their $F_{1}$ hybrids. Zeitshrift für Pflanzenzuchtung, 93, 1-22.

LEGGETT, J. M. 1977. The meiotic behaviour of aneupolyhaploids of the cultivated oat Avena sativa $(2 n=6 x=42)$. Can. J. Gen. Cytol., 19, 651-656.

LEWIS, E. J., HUMPHREYS, M. W. AND CATON, M. P. 1980. Disomic inheritance in Festuca arundinacea Schreb. Zeitschrift für Pflanzenzuchtung, 84, 335-341.

MORGAN, w. G., THOMAS, H. AND LEWIS, E. J. 1988. Cytogenetic studies of hybrids between Festuca gigantea Vill. and Lolium multiflorum Lam. Plant Breeding, 101, 335-343.

RAJHATHY, T. AND THOMAS, H. 1972. Genetic control of chromosome pairing in hexaploid oats. Nature New Biology, 239, 217-219.

RILEY, R. 1960. The diploidisation of polyploid wheat. Heredity, 15, 407-429.

SEARS, E. R. 1976. Genetic control of chromosome pairing in wheat. Ann. Rev. Genet., 10, 31-51.

THOMAS, HUGH, MORGAN, w. G., BORRILL, M. AND EVANS, M. 1982. Meiotic behaviour in polyploid species of Festuca. In: Brandham, P. E. and Bennett, M. D. (eds) Kew Chromosome Conference II. George Allen and Unwin, London, pp. 133-138.

THOMAS, HUW, M. 1990. Analysis of synaptonemal complexes in the amphidiploid of Lolium multiflorum $\times$ Festuca drymeja. Genome, 33, 903-907.

THOMAS, HUW, M. AND MORGAN, w. G. 1990. Analysis of synaptonemal complexes and chromosome pairing at metaphase $I$ in the diploid intergenetic hybrid Lolium multiflorum $\times$ Festuca drymeja. Genome, 33, 465-471. 